\title{
Cardiovascular mechanobiology-a Special Issue to look at the state of the art and the newest insights into the role of mechanical forces in cardiovascular development, physiology and disease
}

\author{
Pamela Swiatlowska $^{1} \cdot$ Thomas Iskratsch $^{1}$ D
}

Received: 31 August 2021 / Accepted: 3 September 2021 / Published online: 11 September 2021

( ) International Union for Pure and Applied Biophysics (IUPAB) and Springer-Verlag GmbH Germany, part of Springer Nature 2021

\begin{abstract}
There has been much progress recently in the area of cardiovascular mechanobiology and this Special Issue aims at taking stock. This editorial gives context of the main motivation for this special issue as well as a brief summary of its content.
\end{abstract}

Cardiovascular diseases (CVD) are the primary cause of mortality, and the disease burden is projected to grow because of sedentary lifestyles and an ageing society. Vascular diseases and especially atherosclerosis, if untreated, are the dominant underlying cause of CVD. Moreover, cardiac disease progressing to heart failure is the leading cause of death among cardiovascular diseases. Underlying genetic causes and mechanisms are progressively being uncovered, e.g. through Genome-Wide Association Studies (GWAS) (Lahm et al. 2021; Shah et al. 2020), metabolomics (Albert and Tang 2018; Wang et al. 2019), proteomics (Lam et al. 2016; Mokou et al. 2017), (single cell) genomics (Samad and Wu 2021) or multi-omics studies (Joshi et al. 2021; Li et al. 2020). However, there is still a gap in understanding that cannot be filled without considering the mechanical/ physical signals and mechanisms in addition to genetic and chemical factors (Iskratsch et al. 2014; Ward and Iskratsch 2020).

Consequentially, there has been a surge in studies investigating the role of mechanical signals in the formation of the heart, or the onset and development of cardiovascular disease. The growing number of research groups dedicated to studying mechanical forces and mechanosensing mechanisms in the cardiovascular system suggests a further expansion in this area and gives hope for novel therapeutic approaches based on the research outcomes.

Thomas Iskratsch

t.iskratsch@qmul.ac.uk

1 School of Engineering and Materials Science, Queen Mary University of London, London, UK
This Special Issue contains a series of review articles that discuss matters from engineering approaches, over computational methods, to clinical implications, to cover all important aspects of cardiovascular mechanobiology. It aims to update on the state of the art and the newest insights of this topic, especially since over the past decades it has become ever clearer that tissue mechanics regulate cardiac function and at the same time contribute to cardiac disease (also outlined in the introductory commentary by Michael Sheetz 2021). Emig et al. (2021) discuss in their review the important parameters and methods to assess especially passive tissue mechanics in vitro or in vivo, while Swiatlowska and Iskratsch (2021) provide an up to date overview of techniques used to study and modulate the cardiovascular mechanobiology across the different scales, from single molecule to whole tissue scale. Tissue mechanics is determined by both the extracellular matrix and intracellular components. The former is discussed in the review from Singh and Young (2021), in which the authors focus on the cardiac nanoenvironment, interacting cardiomyocytes and platforms to study the nanoscale interactions. On the other hand, Crocini and Gotthardt (2021) comprehensively review the intracellular side: especially the role of the sarcomere in active and passive tissue mechanics as well as its potential as a target for therapeutic approaches for cardiac disease. The article from van der Pijl et al. (2021) further explores the N2B and N2A regions of the giant protein titin as major biomechanical and metabolic signalling hubs, in health and disease. Solis and Russell (2021) expand to discuss how deformation of striated muscle proteins due to physical forces and structural changes downstream of chemical signals are both integrated in muscle adaptation. 
Sarcomere-generated forces are transmitted to neighbouring cells through highly organized intercalated discs (ID) located at the cell ends, which are also mechanosignalling hubs. Desmosomes that constitute an integral part of the IDs are further discussed by Zhang et al. (2021), bringing our attention to its non-canonical functions, such as the desmosomal cross-talk with electrical channels, link to protein degradation and inflammation.

On the whole organ level, MacDonald and Quinn (2021) look at how cardiac pacemaking is regulated through sinoatrial node mechanosensitivity, 'membrane' and 'calcium-clocks'.

Not only the scale but also study models have been of great interest among the cardiac mechanobiology field. Induced pluripotent stem cells (iPSC) cardiomyocytes are routinely used in the lab environment and are a promising model system for drug testing, especially in the form of microtissues. However, obtaining mature iPSC cardiomyocytes still constitutes a major problem, which can be improved by precisely controlling the mechanical signals the cells are exposed to. An up to date overview of the different strategies is put together here by Carlos-Oliveira et al. (2021).

With current progress in cardiac computational modelling techniques and future developments, this approach has been applied more broadly along with biological experimental data sets. Sharifi et al. (2021) elegantly outline computational models that simulate cardiac growth and myofiber remodelling, complementing the article with limitations and future perspectives.

Mechanoregulation is also a widely studied topic in the circulatory system. Wang and Valdez-Jasso (2021) take a closer look at the mechanical regulation in pulmonary arterial hypertension, thoroughly discussing recent studies of endothelial cells, smooth muscle cells and fibroblasts in vitro, in vivo and in silico. The review from Johnson et al. (2021) on the other hand discusses how mechanical forces affect the arterial smooth muscle cell cytoskeleton, which eventually projects on cellular phenotype and contractility. From a bioengineering perspective, Nguyen et al. (2021) provide a detailed review of microfluidic platforms for studying the mechanobiology of human circulatory system, while Herault et al. (2021) look at the role of miRNAs and their regulation into clusters and families in vascular mechanobiology.

Fitting to the topic of cardiovascular biology, this issue further includes a self-portrait of the journal Editor Elisabeth Ehler as part of the 'meet the editor' series, in which she discusses her scientific upbringing, source of interest in (cardiac) muscle biology and her thoughts on mentoring and equality(Ehler 2021).

We would like to thank all the authors of this Special Issue for their commitment, efforts and promptness in writing these exciting and timely reviews. We hope that together these will be helpful to everyone working in this area and further enhance the appreciation of the importance of cardiovascular mechanobiology in general.

\section{Declarations}

Conflict of interest The authors declare no competing interests.

\section{References}

Albert CL, Tang WHW (2018) Metabolic biomarkers in heart failure. Heart Fail Clin 14(1):109-118. https://doi.org/10.1016/j.hfc.2017. 08.011

Carlos-Oliveira M, Lozano-Juan F, Occhetta P, Visone R, Rasponi M (2021) Current strategies of mechanical stimulation for maturation of cardiac microtissues. Biophys Rev 13:5. https://doi.org/10. 1007/s12551-021-00841-6

Crocini C, Gotthardt M (2021) Cardiac sarcomere mechanics in health and disease. Biophys Rev 13:5. https://doi.org/10.1007/ s12551-021-00840-7

Ehler E (2021) Biophysical reviews "Meet the Editor Series"Elisabeth Ehler. Biophys Rev 13:5. https://doi.org/10.1007/ s12551-021-00830-9

Emig R, Zgierski-Johnston CM, Timmermann V, Taberner AJ, Nash MP, Kohl P, Peyronnet R (2021) Passive myocardial mechanical properties: meaning, measurement, models. Biophys Rev 13:5. https://doi.org/10.1007/s12551-021-00838-1

Herault S, Naser J, Carassiti D, Chooi KY, Nikolopoulou R, Font ML, Patel MB, Pedrigi R, Krams R (2021) Mechanosensitive pathways are regulated by mechanosensitive miRNA clusters in endothelial cells. Biophys Rev 13:5. https://doi.org/10.1007/ s12551-021-00839-0

Iskratsch T, Wolfenson H, Sheetz MP (2014) Appreciating force and shape-the rise of mechanotransduction in cell biology. Nat Rev Mol Cell Biol 15(12):825-833. https://doi.org/10.1038/nrm3903

Johnson RT, Solanki R, Warren DT (2021) Mechanical programming of arterial smooth muscle cells in health and ageing. Biophys Rev 13:5. https://doi.org/10.1007/s12551-021-00833-6

Joshi A, Rienks M, Theofilatos K, Mayr M (2021) Systems biology in cardiovascular disease: a multiomics approach. Nat Rev Cardiol 18(5):313-330. https://doi.org/10.1038/s41569-020-00477-1

Lahm H, Jia M, Dressen M, Wirth F, Puluca N, Gilsbach R, Keavney BD, Cleuziou J, Beck N, Bondareva O et al (2021) Congenital heart disease risk loci identified by genome-wide association study in European patients. J Clin Invest 131:2. https://doi.org/ 10.1172/JCI141837

Lam MP, Ping P, Murphy E (2016) Proteomics research in cardiovascular medicine and biomarker discovery. J Am Coll Cardiol 68(25):2819-2830. https://doi.org/10.1016/j.jacc.2016.10.031

Li M, Parker BL, Pearson E, Hunter B, Cao J, Koay YC, Guneratne O, James DE, Yang J, Lal S et al (2020) Core functional nodes and sex-specific pathways in human ischaemic and dilated cardiomyopathy. Nat Commun 11(1):2843. https://doi.org/10.1038/ s41467-020-16584-Z

MacDonald EA, Quinn AT (2021) What keeps us ticking? Sinoatrial node mechano-sensitivity: the grandfather-clock of cardiac rhythm. Biophys Rev 13:5. https://doi.org/10.1007/ s12551-021-00831-8 
Mokou M, Lygirou V, Vlahou A, Mischak H (2017) Proteomics in cardiovascular disease: recent progress and clinical implication and implementation. Expert Rev Proteomics 14(2):117-136. https:// doi.org/10.1080/14789450.2017.1274653

Nguyen N, Thurgood P, Sekar NC, Chen S, Pirogova E, Peter K, Baratchi S, Khoshmanesh K (2021) Microfluidic models of the human circulatory system: versatile platforms for exploring mechanobiology and disease modeling. Biophys Rev 13:5. https://doi. org/10.1007/s12551-021-00815-8

Samad T, Wu SM (2021) Single cell RNA sequencing approaches to cardiac development and congenital heart disease. Semin Cell Dev Biol. https://doi.org/10.1016/j.semcdb.2021.04.023

Shah S, Henry A, Roselli C, Lin H, Sveinbjornsson G, Fatemifar G, Hedman AK, Wilk JB, Morley MP, Chaffin MD et al (2020) Genome-wide association and Mendelian randomisation analysis provide insights into the pathogenesis of heart failure. Nat Commun 11(1):163. https://doi.org/10.1038/s41467-019-13690-5

Sharifi H, Mann CK, Rockward AL, Mehri M, Mojumder J, Lee L-C, Campbell KS, Wenk JF (2021) Multiscale simulations of left ventricular growth and remodeling. Biophys Rev 13:5. https://doi.org/ 10.1007/s12551-021-00826-5

Sheetz M (2021) Mechanobiology in cardiac mechanics. Biophys Rev 13:5. https://doi.org/10.1007/s12551-021-00827-4

Singh JP, Young JL (2021) The cardiac nanoenvironment: form and function at the nanoscale. Biophys Rev 13:5. https://doi.org/10. 1007/s12551-021-00834-5

Solis C, Russell B (2021) Striated muscles proteins are regulated both by mechanical deformation and by chemical post-translational modification. Biophys Rev 13:5. https://doi.org/10.1007/ s12551-021-00835-4
Swiatlowska P, Iskratsch T (2021) Tools for studying cardiomyocyte mechanics and mechanosensing across the scales. Biophys Rev 13:5. https://doi.org/10.1007/s12551-021-00837-2

van der Pijl RJ, Domenighetti AA, Sheikh F, Ehler E, Ottenheijm CAC, Lange S (2021) The titin N2B and N2A regions: biomechanical and metabolic signaling hubs in cross-striated muscles. Biophys Rev 13:5. https://doi.org/10.1007/s12551-021-00836-3

Wang A, Valdez-Jasso D (2021) Cellular mechanosignaling in pulmonary arterial hypertension. Biophys Rev 13:5. https://doi.org/10. 1007/s12551-021-00828-3

Wang Z, Zhu C, Nambi V, Morrison AC, Folsom AR, Ballantyne CM, Boerwinkle E, Yu B (2019) Metabolomic pattern predicts incident coronary heart disease. Arterioscler Thromb Vasc Biol 39(7):1475-1482. https://doi.org/10.1161/ATVBAHA.118. 312236

Ward M, Iskratsch T (2020) Mix and (mis-)match - the mechanosensing machinery in the changing environment of the developing, healthy adult and diseased heart. Biochim Biophys Acta Mol Cell Res 1867(3):118436. https://doi.org/10.1016/j.bbamcr.2019.01. 017

Zhang J, Liang Y, Bradford WH, Sheikh F (2021) Desmosomes: emerging pathways and non-canonical functions in cardiac arrhythmias and disease. Biophys Rev 13:5. https://doi.org/10. 1007/s12551-021-00829-2

Publisher's note Springer Nature remains neutral with regard to jurisdictional claims in published maps and institutional affiliations. 